DESY $93-140$

ISSN $0418-9833$

October 1993

hep-th/9310162

\title{
ON THE PATH INTEGRAL IN IMAGINARY LOBACHEVSKY SPACE
}

\author{
Christian Grosche* \\ II. Institut für Theoretische Physik \\ Universität Hamburg, Luruper Chaussee 149 \\ 22761 Hamburg, Germany
}

\begin{abstract}
The path integral on the single-sheeted hyperboloid, i.e. in $D$-dimensional imaginary Lobachevsky space, is evaluated. A potential problem which we call "Kepler-problem", and the case of a constant magnetic field are also discussed.
\end{abstract}

* Supported by Deutsche Forschungsgemeinschaft under contract number GR 1031/2-1. 



\section{Introduction.}

Motion on spaces with constant curvature, positive as well as negative constant curvature, is of particular interest and appears in several topics in theoretical physics. Let us e.g. mention string theory where the perturbative expansion à la Polyakov [15] leads to the consideration of determinants of Laplacians on Riemann surfaces of arbitrary genus, a theory where the underlying space is the Poincaré, respectively Lobachevsky space, a space of constant negative curvature.

Another example is the Kepler problem in spaces of constant curvature [2, 18]. Here one is interested in the comparison of the symmetry properties of this problem, where one e.g. finds that the coordinate systems which separate the Kepler problem in spaces of constant curvature are only two, namely the (pseudo-) spherical and the (pseudo-) conical, whereas in flat space there are four [32].

The evaluations of propagators and its short-time behaviour, and Green functions are also important in cosmological models. They appear in several models derived from the WheelerDeWitt equation and quantum gravity, respectively, and lead in a natural way to models, respectively spaces, with constant curvature. As the simplest case one can study the free motion in these spaces. Here several models can appear in the case of constant negative curvature: The single-sheeted and the two-sheeted hyperboloid. Most simply, they are studied in the twodimensional case. The two-sheeted hyperboloid is a particular realization of the Poincaré plane, where only one sheet has been taken, whereas the single-sheeted has different properties and has not been studied in such great detail as the former one. However, some contributions exist, mainly by Gel'fand, Graev and Vilenkin who call this space imaginary Lobachevsky space [11, 12, 35] and have studied its geometrical structure and group theoretical properties. It has the peculiarity that the distance $r$ of two points defined by $\cosh k r$ ( $k$ is the curvature) may be positive and imaginary because $\cosh k r \in[0, \infty)$, i.e. it is a space-like set, in comparison to the usual two-sheeted hyperboloid, also called "pseudosphere", which is a time-like set. From the point of view of special relativity, Lobachevskian models are of interest because the velocity space, say, possess a constant negative curvature (equal to $1 / c^{2}$ ), and the single-sheeted hyperboloid in particular corresponds to the unphysical region of the variables.

In this note I want to study the path integral on the (D-1)-dimensional single-sheeted hyperboloid, denoted in the following by $\mathcal{H}_{-1}^{(D)}$ which is done in Section 2. This special system was not subject to path integration until now. The path integral on the pseudosphere has been intensively studied in Refs. [3, 18, 20, 24], with its higher dimensional generalizations in [22, 26]. In comparison to this usual pseudosphere we will find that in the case of $\mathcal{H}_{-1}^{(D)}$ bound states can appear, depending on the angular momentum number, which is not possible for the quantum motion on the pseudosphere.

As we will see in Section 3, also a potential problem on the single-sheeted hyperboloid can be discussed, which will be called "Kepler-problem" on the single-sheeted hyperboloid.

In Section 4, the case of a constant magnetic field on $\mathcal{H}_{-1}^{(D)}$ will be discussed, Section 5 contains some concluding remarks, and in the Appendix the path integral identity for the modified PöschlTeller potential is given.

\section{The Path Integral.}

For simplicity we first consider the simplest case, i.e. $D=3$. We start with the equation for the two-dimensional single-sheeted hyperboloid $\mathcal{H}_{-1}^{(3)}(1 / k=R>0)$

$$
(\vec{x}, \vec{x})=x_{0}^{2}-x_{1}^{2}-x_{2}^{2}=-R^{2} .
$$


We introduce pseudo-spherical polar coordinates on $\mathcal{H}_{-1}^{(3)}$

$$
x_{0}=R \sinh \tau, \quad x_{1}=R \cosh \tau \sin \phi, \quad x_{2}=R \cosh \tau \cos \phi,
$$

where $\tau \in(-\infty, \infty)$ and $\phi \in[0,2 \pi)$. The addition theorem on the single-sheeted hyperboloid $\mathcal{H}_{-1}^{(3)}$ has the form $\left(\vec{x}, \vec{y} \in \mathcal{H}_{-1}^{(3)}\right)$

$$
\begin{aligned}
\cosh k r & =\frac{\vec{x}_{1} \cdot \vec{x}_{2}}{\left|\vec{x}_{1}\right| \cdot\left|\vec{x}_{2}\right|} \\
& =\left(\sinh \tau_{1} \sinh \tau_{2}-\cosh \tau_{1} \cosh \tau_{2} \cos \left(\phi_{2}-\phi_{1}\right)\right) .
\end{aligned}
$$

We find for the metric: $\left(g_{a b}\right)=R^{2} \operatorname{diag}\left(1,-\cosh ^{2} \tau\right)$, and therefore $g=\sqrt{\left|\operatorname{det}\left(g_{a b}\right)\right|}=R^{2} \cosh \tau$. According to the canonical formalism [5, 9, 16, 25, 27] we construct the path integral on $\mathcal{H}_{-1}^{(3)}$ as follows $\left(T=t^{\prime \prime}-t^{\prime}\right)$

$$
\begin{aligned}
K^{\left(\mathcal{H}_{-1}^{(3)}\right)}\left(x_{1}^{\prime \prime}, x_{1}^{\prime}, x_{2}^{\prime \prime}, x_{2}^{\prime}, x_{3}^{\prime \prime}, x_{3}^{\prime} ; T\right) \equiv K^{\left(\mathcal{H}_{-1}^{(3)}\right)}\left(\tau^{\prime \prime}, \tau^{\prime}, \phi^{\prime \prime}, \phi^{\prime} ; T\right) \\
=\int_{x_{1}\left(t^{\prime}\right)=x_{1}^{\prime}}^{x_{1}\left(t^{\prime \prime}\right)=x_{1}^{\prime \prime}} \mathcal{D} x_{1}(t) \int_{\left.x_{2} t^{\prime}\right)=x_{2}^{\prime}}^{x_{2}\left(t^{\prime \prime}\right)=x_{2}^{\prime \prime}} \mathcal{D} x_{2}(t) \int_{x_{3}\left(t^{\prime}\right)=x_{3}^{\prime}}^{x_{3}\left(t^{\prime \prime}\right)=x_{3}^{\prime \prime}} \mathcal{D} x_{3}(t) \exp \left[\frac{\mathrm{i} m}{2 \hbar} \int_{t^{\prime}}^{t^{\prime \prime}}\left(\dot{x}_{1}^{2}-\dot{x}_{2}^{2}-\dot{x}_{3}^{2}\right) d t\right] \\
\rightarrow \frac{1}{R^{2}} \lim _{N \rightarrow \infty}\left(\frac{m R^{2}}{2 \pi \epsilon \hbar}\right) \prod_{j=1}^{N-1} \int_{-\infty}^{\infty} \cosh \tau_{j} d \tau_{j} \int_{0}^{2 \pi} d \phi_{j} \\
\quad \times \exp \left\{\frac{\mathrm{i}}{\hbar} \sum_{j=1}^{N}\left[\frac{m R^{2}}{2 \epsilon}\left(\Delta^{2} \tau_{j}-\widehat{\cosh }^{2} \tau_{j} \Delta^{2} \phi_{j}\right)-\frac{\epsilon \hbar^{2}}{8 m R^{2}}\left(1+\frac{1}{\cosh ^{2} \tau_{j}}\right)\right]\right\} \\
\equiv \frac{1}{R^{2}} \int_{\tau\left(t^{\prime}\right)=\tau^{\prime}} \cosh \tau \mathcal{D} \tau(t) \int_{\phi\left(t^{\prime \prime}\right)=\phi^{\prime \prime}} \mathcal{D} \phi(t) \\
\quad \times \exp \left\{\frac{\mathrm{i}}{\hbar} \int_{t^{\prime}}^{t^{\prime \prime}}\left[\frac{m}{2} R^{2}\left(\dot{\tau}^{2}-\cosh ^{2} \tau \dot{\phi}^{2}\right)-\frac{\hbar^{2}}{8 m R^{2}}\left(1+\frac{1}{\cosh ^{2} \tau}\right)\right] d t\right\} .
\end{aligned}
$$

Here are $\epsilon=T / N, q_{j}=q\left(t^{\prime}+j \epsilon\right), \widehat{f}^{2}\left(q_{j}\right)=f\left(q_{j}\right) f\left(q_{j-1}\right)$ for any function of the coordinates $\tau$ and $\phi$, and $j=0, \ldots, N, \Delta q_{j}=q_{j}-q_{j-1}$, and we interpret the limit $N \rightarrow \infty$ as equivalent with $\epsilon \rightarrow 0$, $T$ fixed. Note that due to the indefinite metric the factors "i" in the "measure term" cancel each other [3]. The corresponding short-time propagator is given by

$$
\begin{aligned}
& K^{\left(\mathcal{H}_{-1}^{(3)}\right)}\left(\tau_{j}, \tau_{j-1}, \phi_{j}, \phi_{j-1} ; \epsilon\right)=\frac{m}{2 \pi \epsilon \hbar} \cosh \tau_{j} \\
& \quad \times \exp \left\{\frac{\mathrm{i}}{\hbar}\left[\frac{m R^{2}}{2 \epsilon}\left(\Delta^{2} \tau_{j}-\widehat{\cosh }^{2} \tau_{j} \Delta^{2} \phi_{j}\right)-\frac{\epsilon \hbar^{2}}{8 m R^{2}}\left(1+\frac{1}{\cosh ^{2} \tau_{j}}\right)\right]\right\} .
\end{aligned}
$$

Note that the pre-exponential factor does not depend on $R$. The $\phi$-path integration can be separated [21] immediately and we obtain

$$
K^{\left(\mathcal{H}_{-1}^{(3)}\right)}\left(\tau^{\prime \prime}, \tau^{\prime}, \phi^{\prime \prime}, \phi^{\prime} ; T\right)=\frac{\mathrm{e}^{-\mathrm{i} \hbar T / 8 m R^{2}}}{\left(-\cosh \tau^{\prime} \cosh \tau^{\prime \prime}\right)^{1 / 2}} \sum_{l=-\infty}^{\infty} \frac{\mathrm{e}^{\mathrm{i} l\left(\phi^{\prime \prime}-\phi^{\prime}\right)}}{2 \pi R^{2}} K_{l}^{\left(\mathcal{H}_{-1}^{(3)}\right)}\left(\tau^{\prime \prime}, \tau^{\prime} ; T\right)
$$

with $K_{l}^{\left(\mathcal{H}_{-1}^{(3)}\right)}\left(\tau^{\prime \prime}, \tau^{\prime} ; T\right)$ given by

$$
K_{l}^{\left(\mathcal{H}_{-1}^{(3)}\right)}\left(\tau^{\prime \prime}, \tau^{\prime} ; T\right)=\int_{\tau\left(t^{\prime}\right)=\tau^{\prime}}^{\tau\left(t^{\prime \prime}\right)=\tau^{\prime \prime}} \mathcal{D} \tau(t) \exp \left\{\frac{\mathrm{i}}{\hbar} \int_{t^{\prime}}^{t^{\prime \prime}}\left[\frac{m}{2} R^{2} \dot{\tau}^{2}+\frac{\hbar^{2}}{2 m R^{2}} \frac{l^{2}-1 / 4}{\cosh ^{2} \tau}\right] d t\right\},
$$


which is a usual one-dimensional path integral. This path integral has the form of the special case of the modified Pöschl-Teller potential as sketched in the Appendix. Therefore we can write down the solution of the path integral on the single-sheeted hyperboloid $\left(n=0,1, \ldots, N_{M}<|l|-\frac{1}{2}\right)$

$$
\begin{aligned}
& \frac{1}{R^{2}} \frac{\mathrm{i}}{\hbar} \int_{0}^{\infty} d T \mathrm{e}^{\mathrm{i} T E / \hbar} \int_{\tau\left(t^{\prime}\right)=\tau^{\prime}}^{\tau\left(t^{\prime \prime}\right)=\tau^{\prime \prime}} \cosh \tau \mathcal{D} \tau(t) \int_{\phi\left(t^{\prime}\right)=\phi^{\prime}}^{\phi\left(t^{\prime \prime}\right)=\phi^{\prime \prime}} \mathcal{D} \phi(t) \\
& \times \exp \left\{\frac{\mathrm{i}}{\hbar} \int_{t^{\prime}}^{t^{\prime \prime}}\left[\frac{m}{2} R^{2}\left(\dot{\tau}^{2}-\cosh ^{2} \tau \dot{\phi}^{2}\right)-\frac{\hbar^{2}}{8 m R^{2}}\left(1+\frac{1}{\cosh ^{2} \tau}\right)\right] d t\right\} \\
& =\left(-\cosh \tau^{\prime} \cosh \tau^{\prime \prime}\right)^{-1 / 2} \sum_{l=-\infty}^{\infty} \frac{\mathrm{e}^{\mathrm{i} l\left(\phi^{\prime \prime}-\phi^{\prime}\right)}}{2 \pi} \\
& \times \frac{m}{\hbar^{2}} \Gamma\left(\sqrt{-\frac{2 m R^{2} E}{\hbar^{2}}+\frac{1}{4}}-|l|+\frac{1}{2}\right) \Gamma\left(\sqrt{-\frac{2 m R^{2} E}{\hbar^{2}}+\frac{1}{4}}+|l|+\frac{1}{2}\right) \\
& \times P_{|l|-1 / 2}^{-\sqrt{-2 m R^{2} E / \hbar^{2}+1 / 4}}\left(\tanh \tau_{<}\right) P_{|l|-1 / 2}^{-\sqrt{-2 m R^{2} E / \hbar^{2}+1 / 4}}\left(-\tanh \tau_{>}\right) \\
& =\left(-\cosh \tau^{\prime} \cosh \tau^{\prime \prime}\right)^{-1 / 2} \sum_{l=-\infty}^{\infty} \frac{\mathrm{e}^{\mathrm{i} l\left(\phi^{\prime \prime}-\phi^{\prime}\right)}}{2 \pi R^{2}} \\
& \times\left[\sum_{n=0}^{N_{M}}\left(n-|l|-\frac{1}{2}\right) \frac{\Gamma(2|l|-n)}{n !} \frac{P_{|l|-1 / 2}^{n-|l|+\frac{1}{2}}\left(\tanh \tau^{\prime}\right) P_{|l|-1 / 2}^{n-|l|+\frac{1}{2}}\left(\tanh \tau^{\prime \prime}\right)}{-\hbar^{2}\left[\left(n-|l|+\frac{1}{2}\right)^{2}-1 / 4\right] / 2 m R^{2}-E}\right. \\
& \left.+\frac{1}{2} \int_{-\infty}^{\infty} \frac{d p p \sinh \pi p}{\hbar^{2}\left(p^{2}+1 / 4\right) / 2 m R^{2}-E} \frac{P_{|l|-1 / 2}^{\mathrm{i} p}\left(\tanh \tau^{\prime \prime}\right) P_{|l|-1 / 2}^{-\mathrm{i} p}\left(\tanh \tau^{\prime}\right)}{\cos ^{2} \pi l+\sinh ^{2} \pi p}\right] .
\end{aligned}
$$

Wave-functions and energy spectrum are easily read off from the spectral expansion (12). Note that for $l \neq 0$ there are bound states. The generalization to higher dimensions can be done in a straightforward way, by replacing the circular wave-functions by the hyperspherical harmonics $S_{l}^{\mu}(\Omega)$, and the quantum number $l \in \mathbb{Z}$ by the corresponding principle quantum number $l \in \mathbb{N}_{0}$, including the appropriate changes in the effective Lagrangian, and with the prefactor replaced by $R^{1-D}$. In order to to this we introduce the (pseudo-bispherical) coordinate system [3, 11, 12, 35]

$$
\begin{aligned}
& x_{0}=R \sinh \tau, \\
& x_{1}=R \cosh \tau \cos \theta_{D-2}, \\
& x_{2}=R \cosh \tau \sin \theta_{D-2} \cos \theta_{D-3}, \\
& \quad \vdots \\
& x_{D-2}=R \cosh \tau \sin \theta_{D-2} \ldots \cos \theta_{2} \cos \phi, \\
& x_{D-1}=R \cosh \tau \sin \theta_{D-2} \ldots \cos \theta_{2} \sin \phi,
\end{aligned}
$$

where $\tau \in(-\infty, \infty), \theta_{1} \equiv \phi \in[0,2 \pi)$, and $\theta_{k} \in[0, \pi), k=2, \ldots, D-2$. The metric tensor on the (D-1)-dimensional single-sheeted hyperboloid is given by: $\left(g_{a b}\right)=R^{2} \operatorname{diag}\left(1,-\cosh ^{2} \tau,-\cosh ^{2} \tau\right.$ $\left.\times \sin ^{2} \theta_{D-2}, \ldots,-\cosh ^{2} \tau \ldots \sin ^{2} \theta_{2}\right)(a, b=1, \ldots, D-1)$. Therefore we obtain for the Hamiltonian on $\mathcal{H}_{-1}^{(D)}$

$$
\begin{aligned}
H= & -\frac{\hbar^{2}}{2 m R^{2}}\left\{\left[\frac{\partial^{2}}{\partial \tau^{2}}+(D-2) \tanh \tau \frac{\partial}{\partial \tau}\right]\right. \\
& \left.-\frac{1}{\cosh ^{2} \tau}\left[\frac{\partial^{2}}{\partial \theta_{D-2}^{2}}+(D-3) \operatorname{coth} \theta_{D-2} \frac{\partial}{\partial \theta_{D-2}}\right]-\ldots-\frac{1}{\cosh ^{2} \tau \ldots \sin ^{2} \theta_{2}} \frac{\partial^{2}}{\partial \phi}\right\}
\end{aligned}
$$




$$
=\frac{1}{2 m R^{2}}\left[p_{\tau}^{2}-\frac{1}{\cosh ^{2} \tau} p_{\theta_{D-2}}^{2}-\ldots-\frac{1}{\cosh ^{2} \tau \ldots \sin ^{2} \theta_{2}} p_{\phi}^{2}\right]+\Delta V(\tau,\{\theta\}),
$$

with the quantum potential

$$
\Delta V(\tau,\{\theta\})=\frac{\hbar^{2}}{8 m R^{2}}\left[(D-2)^{2}+\frac{1}{\cosh ^{2} \tau}+\ldots+\frac{1}{\cosh ^{2} \tau \ldots \sin ^{2} \theta_{2}}\right]
$$

$\left[\{\theta\}\right.$ denotes the set of variables $\left.\theta_{k}(k=1, \ldots, D-2)\right]$. Furthermore $\left(g=\operatorname{det}\left(g_{a b}\right)=\cosh ^{D-2} \tau\right.$ $\left.\times \prod_{k=1}^{D-2}\left(\sin \theta_{k}\right)^{k-1}\right)$

$$
p_{a}=\frac{\hbar}{\mathrm{i}}\left(\frac{\partial}{\partial q^{a}}+\frac{\Gamma_{a}}{2}\right), \quad \Gamma_{a}=\frac{\partial \log \sqrt{g}}{\partial q^{a}} .
$$

Thus we obtain for the (Lagrangian) path integral on $\mathcal{H}_{-1}^{(D)}\left[\vec{x}=\left(x_{0}, \ldots, x_{D-1}\right)\right]$

$$
\begin{aligned}
& K^{\left(\mathcal{H}_{-1}^{(D)}\right)}\left(\vec{x}^{\prime \prime}, \vec{x}^{\prime} ; T\right) \equiv K^{\left(\mathcal{H}_{-1}^{(D)}\right)}\left(\tau^{\prime \prime}, \tau,\left\{\theta^{\prime \prime}\right\},\left\{\theta^{\prime}\right\} ; T\right) \\
& =\prod_{k=0}^{D-1} \int_{x_{k}\left(t^{\prime}\right)=x_{k}^{\prime}}^{x_{k}\left(t^{\prime \prime}\right)=x_{k}^{\prime \prime}} \mathcal{D} x_{k}(t) \exp \left[\frac{\mathrm{i} m}{2 \hbar} \int_{t^{\prime}}^{t^{\prime \prime}}\left(\dot{x}_{0}^{2}-\sum_{k=1}^{D-1} \dot{x}_{k}^{2}\right) d t\right] \\
& \rightarrow \int_{\tau\left(t^{\prime}\right)=\tau^{\prime}}^{\tau\left(t^{\prime \prime}\right)=\tau^{\prime \prime}} \cosh ^{D-2} \tau \mathcal{D} \tau(t) \int_{\Omega\left(t^{\prime}\right)=\Omega^{\prime}} \mathcal{D} \Omega(t) \exp \left\{\frac{\mathrm{i}}{\hbar} \int_{t^{\prime}}^{t^{\prime \prime}}\left[\mathcal{L}_{C l}(\tau, \dot{\tau},\{\theta\},\{\dot{\theta}\})-\Delta V(\tau,\{\theta\})\right]\right\} \\
& =R^{1-D} \lim _{N \rightarrow \infty}\left(\frac{m R^{2}}{2 \pi \mathrm{i} \epsilon \hbar}\right)^{\frac{N}{2}(D-2)}\left(\frac{\mathrm{i} m R^{2}}{2 \pi \hbar \epsilon}\right)^{\frac{N}{2}} \prod_{j=1}^{N-1} \int_{0}^{\infty} \cosh ^{D-2} \tau_{j} d \tau_{j} \int d \Omega_{j} \\
& \quad \times \exp \left\{\frac{\mathrm{i}}{\hbar} \sum_{j=1}^{N}\left[\mathcal{L}_{C l}^{N}\left(\tau_{j-1}, \tau_{j},\left\{\theta_{j-1}\right\},\left\{\theta_{j}\right\}\right)-\epsilon \Delta V\left(\tau_{j},\left\{\theta_{j}\right\}\right)\right]\right\} .
\end{aligned}
$$

$\mathcal{L}_{C l}$ is the classical Lagrangian

$$
\mathcal{L}_{C l}(\tau, \dot{\tau},\{\theta\},\{\dot{\theta}\})=\frac{m R^{2}}{2}\left[\dot{\tau}^{2}-\cosh ^{2} \tau \dot{\theta}_{D-2}^{2}-\ldots-\left(\cosh ^{2} \tau \ldots \sin ^{2} \theta_{2}\right) \dot{\phi}^{2}\right],
$$

and its counterpart on the lattice reads

$$
\begin{aligned}
& \mathcal{L}_{C l}^{N}\left(\tau_{j-1}, \tau_{j},\left\{\theta_{j-1}\right\},\left\{\theta_{j}\right\}\right) \\
& =\frac{m R^{2}}{2 \epsilon^{2}}\left[\Delta^{2} \tau_{j}-\widehat{\cosh ^{2}} \tau_{j} \Delta^{2} \theta_{D-2, j}-\ldots-\left(\widehat{\cosh ^{2}} \tau_{j} \ldots \widehat{\sin }^{2} \theta_{2, j}\right) \Delta^{2} \phi_{j}\right] .
\end{aligned}
$$

$d \Omega=\prod_{k=1}^{D-2}\left(\sin \theta_{k}\right)^{k-1} d \theta_{k}$ is the $(D-2)$-dimensional surface element on the unit-sphere $S^{(D-2)}$. Note again that the pre-exponential factor in the short-time kernel does not depend on $R$.

Due to the very singular nature of $\Delta V(\tau,\{\theta\})$ this path integral is at it stands not tractable. However, we can use a path integral identity (based on a method developed in [13, 33]) already derived in [25, 26] to simplify the path integration significantly and separate the angular variables $\theta_{D-2}, \ldots, \phi$ from the hyperbolic coordinate $\tau$. I introduce the quantity $\psi^{\left({ }^{\prime},{ }^{\prime \prime}\right)}$ defined by

$$
\cos \psi^{\left({ }^{\prime \prime},{ }^{\prime}\right)}=\cos \theta_{D-2}^{\prime} \cos \theta_{D-2}^{\prime \prime}+\sum_{m=1}^{D-3} \cos \theta_{m}^{\prime} \cos \theta_{m}^{\prime \prime} \prod_{n=m+1}^{D-2} \sin \theta_{n}^{\prime \prime} \sin \theta_{n}^{\prime \prime}+\prod_{n=1}^{D-2} \sin \theta_{n}^{\prime} \sin \theta_{n}^{\prime \prime},
$$

which is actually the addition theorem on the $S^{(D-2)}$-sphere and $\cos \psi^{\left({ }^{\prime},{ }^{\prime \prime}\right)}=\Omega^{\prime} \cdot \Omega^{\prime \prime}$, where $\Omega^{\left({ }^{\prime},{ }^{\prime \prime}\right)}$ are unit vectors on the $S^{(D-2)}$-sphere. Using the result of [25] the following path integral 
identity can be achieved (replace $R^{2}=r_{j}^{2}=-\cosh ^{2} \tau_{j}$ in $(2.27)$ in [25])

$$
\begin{aligned}
\exp \{- & \left.\frac{\mathrm{i} m R^{2}}{2 \epsilon \hbar} \cosh ^{2} \tau_{j}\left[\Delta^{2} \theta_{D-2, j}+\ldots+\left(\sin ^{2} \widehat{\theta_{D}-2, j} \ldots \widehat{\sin }^{2} \theta_{2, j}\right) \Delta^{2} \phi_{j}\right]\right\} \\
\doteq \exp [- & \frac{\mathrm{i} m R^{2}}{\epsilon \hbar} \widehat{\cosh ^{2}} \tau_{j}\left(1-\cos \psi_{j-1, j}\right) \\
& \left.\quad+\frac{\mathrm{i} \epsilon \hbar}{8 m R^{2} \cosh ^{2} \tau_{j}}\left(1+\frac{1}{\sin ^{2} \theta_{D-2, j}}+\ldots+\frac{1}{\sin ^{2} \theta_{D-2, j} \ldots \sin ^{2} \theta_{2, j}}\right)\right] .
\end{aligned}
$$

Here I have used the symbol $\doteq$ - following DeWitt [5]- to denote "equivalence as far as use in the path integral is concerned". The highly singular terms are cancelling and I obtain

$$
\begin{aligned}
K^{\left(\mathcal{H}_{-1}^{(D)}\right)}\left(\tau^{\prime \prime}, \tau^{\prime},\left\{\theta^{\prime \prime}\right\}\right. & \left.,\left\{\theta^{\prime}\right\} ; T\right)=R^{1-D} \int_{\tau\left(t^{\prime}\right)=\tau^{\prime}}^{\tau\left(t^{\prime \prime}\right)=\tau^{\prime \prime}} \cosh ^{D-2} \tau \mathcal{D} \tau(t) \int_{\Omega\left(t^{\prime}\right)=\Omega^{\prime}}^{\Omega\left(t^{\prime \prime}\right)=\Omega^{\prime \prime}} \mathcal{D} \Omega(t) \\
& \times \exp \left\{\frac{\mathrm{i} m R^{2}}{2 \hbar} \int_{t^{\prime}}^{t^{\prime \prime}}\left[\dot{\tau}^{2}-2 \cosh ^{2} \tau(1-\cos \psi)\right]-\frac{\mathrm{i} \hbar T(D-2)^{2}}{8 m R^{2}}\right\} .
\end{aligned}
$$

Expanding now the exponential according to [14], p.980:

$$
\mathrm{e}^{z \cos \psi^{\left({ }^{\prime \prime}\right)}}=\left(\frac{2}{z}\right)^{\nu} \Gamma(\nu) \sum_{l=0}^{\infty}(l+\nu) C_{l}^{\nu}\left(\cos \psi^{\left({ }^{\prime},,^{\prime \prime}\right)}\right) I_{l+\nu}(z)
$$

where $C_{l}^{\nu}(x)$ is a Gegenbauer-polynomial, together with [7], Chapter XI

$$
\sum_{\mu=1}^{M} S_{l}^{\mu}\left(\Omega^{\prime}\right) S_{l}^{\mu}\left(\Omega^{\prime \prime}\right)=\frac{1}{\Omega(D)} \frac{2 l+D-2}{D-2} C_{l}^{\frac{D-2}{2}}\left(\cos \psi^{\left({ }^{\prime},{ }^{\prime \prime}\right)}\right)
$$

where the $S_{l}^{\mu}(\Omega)$ are the real hyper-spherical harmonics of degree $l$ with unit vector $\Omega$ on the $S^{(D-1)}$-sphere, $l \in \mathbb{N}_{0}, \Omega(D)=2 \pi^{D / 2} / \Gamma(D / 2)$ is the volume of the $D$-dimensional unit-sphere $S^{(D-1)}$, and $\mu=1, \ldots, M, M=(2 l+D-2)(l+D-3) ! / l !(D-3) !$. Thus for $\nu=\frac{D-3}{2}$ in (24), i.e. on $S^{(D-2)}$

$$
\mathrm{e}^{z \cos \psi^{\left({ }^{\prime \prime}\right)}}=2 \pi\left(\frac{2 \pi}{z}\right)^{\frac{D-3}{2}} \sum_{l=0}^{\infty} \sum_{\mu=1}^{M} S_{l}^{\mu}\left(\Omega^{\prime}\right) S_{l}^{\mu}\left(\Omega^{\prime \prime}\right) I_{l+\frac{D-3}{2}}(z)
$$

The angular variables in the path integral on the (D-1)-dimensional single-sheeted hyperboloid $\mathcal{H}_{-1}^{(D)}$ can be therefore separated in a straightforward way and we obtain

$$
\begin{aligned}
& K^{\left(\mathcal{H}_{-1}^{(D)}\right)}\left(\tau^{\prime \prime}, \tau,\left\{\theta^{\prime \prime}\right\},\left\{\theta^{\prime}\right\} ; T\right)=\frac{R^{1-D}}{\left(-\cosh \tau^{\prime} \cosh \tau^{\prime \prime}\right)^{(D-2) / 2}} \\
& \quad \times \sum_{l=0}^{\infty} \sum_{\mu=1}^{M} S_{l}^{\mu}\left(\Omega^{\prime}\right) S_{l}^{\mu}\left(\Omega^{\prime \prime}\right) \exp \left[-\frac{\mathrm{i} \hbar T}{8 m R^{2}}(D-2)^{2}\right] K_{l}^{\left(\mathcal{H}_{-1}^{(D)}\right)}\left(\tau^{\prime \prime}, \tau^{\prime} ; T\right)
\end{aligned}
$$

and $K_{l}^{\left(\mathcal{H}_{-1}^{(D)}\right)}\left(\tau^{\prime \prime}, \tau^{\prime} ; T\right)$ is given by

$$
K_{l}^{\left(\mathcal{H}_{-1}^{(D)}\right)}\left(\tau^{\prime \prime}, \tau^{\prime} ; T\right)=\int_{\tau\left(t^{\prime}\right)=\tau^{\prime}}^{\tau\left(t^{\prime \prime}\right)=\tau^{\prime \prime}} \mathcal{D} \tau(t) \exp \left\{\frac{\mathrm{i}}{\hbar} \int_{t^{\prime}}^{t^{\prime \prime}}\left[\frac{m}{2} R^{2} \dot{\tau}^{2}+\frac{\hbar^{2}}{2 m R^{2}} \frac{\left(l+\frac{D-3}{2}\right)^{2}-1 / 4}{\cosh ^{2} \tau}\right] d t\right\} .
$$


Therefore we obtain similarly as before $\left(\tilde{E}=E-\hbar^{2}(D-2)^{2} / 8 m R^{2}\right)$

$$
\begin{aligned}
& \frac{\mathrm{i}}{\hbar} \int_{0}^{\infty} d T \mathrm{e}^{\mathrm{i} T E / \hbar} K^{\left(\mathcal{H}_{-1}^{(D)}\right)}\left(\left\{\theta^{\prime \prime}\right\},\left\{\theta^{\prime}\right\}, \tau^{\prime \prime}, \tau^{\prime} ; T\right) \\
& =\frac{1}{2} \pi^{\frac{1-D}{2}} \Gamma\left(\frac{D-1}{2}\right) R^{3-D}\left(-\cosh \tau^{\prime} \cosh \tau^{\prime \prime}\right)^{-(D-2) / 2} \sum_{l=0}^{\infty} \frac{2 l+D-3}{D-3} C_{l}^{(D-3) / 2}\left(\cos \psi^{\left({ }^{\prime \prime},{ }^{\prime}\right)}\right) \\
& \quad \times \frac{m}{\hbar^{2}} \Gamma\left(\sqrt{-2 m R^{2} \tilde{E}} / \hbar-l-\frac{D-4}{2}\right) \Gamma\left(\sqrt{-2 m R^{2} \tilde{E}} / \hbar+l+\frac{D-2}{2}\right) \\
& \quad \times P_{l+(D-4) / 2}^{-\sqrt{-2 m R^{2} \tilde{E}} / \hbar}\left(\tanh \tau_{<}\right) P_{l+(D-4) / 2}^{-\sqrt{-2 m R^{2} \tilde{E}} / \hbar}\left(-\tanh \tau_{>}\right) .
\end{aligned}
$$

Let us set $D=2 d+4$ with $d=0,1, \ldots$ Then $\left(l+\frac{D-3}{2}\right)^{2}-1 / 4=(l+d)[(l+d)+1]$ and we see that in this case the radial propagator on $\mathcal{H}_{-1}^{(D)}$ yields the propagator of a reflectionless potential [4]. Hence we can explicitly state for the propagator $(N=l+d)$

$$
\begin{aligned}
& K^{\left(\mathcal{H}_{-1}^{(D)}\right)}\left(\left\{\theta^{\prime \prime}\right\},\left\{\theta^{\prime}\right\}, \tau^{\prime \prime}, \tau^{\prime} ; T\right) \\
& =\frac{R^{1-D}}{\left(-\cosh \tau^{\prime} \cosh \tau^{\prime \prime}\right)^{(D-2) / 2}} \sum_{l=0}^{\infty} \sum_{\mu=1}^{M} S_{l}^{\mu}\left(\Omega^{\prime}\right) S_{l}^{\mu}\left(\Omega^{\prime \prime}\right) \\
& \times\left\{\sum_{n=0}^{N-1} \exp \left[\frac{\mathrm{i} \hbar T}{2 m R^{2}}\left((N-n)^{2}-\frac{(D-2)^{2}}{4}\right)\right]\right. \\
& \times(N-n) \frac{(2 N-n) !}{n !} P_{N}^{n-N}\left(\tanh \tau^{\prime}\right) P_{N}^{n-N}\left(\tanh \tau^{\prime \prime}\right) \\
& \left.+\int_{-\infty}^{\infty} \frac{d p p}{2 \sinh \pi p} \exp \left[-\frac{\mathrm{i} \hbar T}{2 m R^{2}}\left(p^{2}+\frac{(D-2)^{2}}{4}\right)\right] P_{N}^{-\mathrm{i} p}\left(\tanh \tau^{\prime}\right) P_{N}^{\mathrm{i} p}\left(\tanh \tau^{\prime \prime}\right)\right\} \\
& =\frac{1}{2} \pi^{\frac{1-D}{2}} \Gamma\left(\frac{D-1}{2}\right) R^{1-D}\left(-\cosh \tau^{\prime} \cosh \tau^{\prime \prime}\right)^{-(D-2) / 2} \sum_{l=0}^{\infty} \frac{2 l+D-3}{D-3} C_{l}^{(D-3) / 2}\left(\cos \psi^{\left({ }^{\prime \prime},{ }^{\prime}\right)}\right) \\
& \times\left\{\sqrt{\frac{m}{2 \pi \mathrm{i} \hbar T}} \exp \left[-\frac{m R^{2}}{2 \mathrm{i} \hbar T}\left(\tau^{\prime \prime}-\tau^{\prime}\right)^{2}\right]+\frac{1}{2} \sum_{n=0}^{N-1} \exp \left[\frac{\mathrm{i} \hbar T}{2 m R^{2}}\left((N-n)^{2}-\frac{(D-2)^{2}}{4}\right)\right]\right. \\
& \times(N-n) \frac{(2 N-n) !}{n !} P_{N}^{n-N}\left(\tanh \tau^{\prime}\right) P_{N}^{n-N}\left(\tanh \tau^{\prime \prime}\right) \\
& \times\left[\operatorname{erf}\left(\sqrt{\frac{\mathrm{i} \hbar T}{2 m R^{2}}}(N-n)-\left(\tau^{\prime \prime}-\tau^{\prime}\right) \sqrt{\frac{m R^{2}}{2 \mathrm{i} \hbar T}}\right)\right. \\
& \left.\left.+\operatorname{erf}\left(\sqrt{\frac{\mathrm{i} \hbar T}{2 m R^{2}}}(N-n)+\left(\tau^{\prime \prime}-\tau^{\prime}\right) \sqrt{\frac{m R^{2}}{2 \mathrm{i} \hbar T}}\right)\right]\right\},
\end{aligned}
$$

where in the resummation use has been made of the integral representations [14], p.497, $\Re \beta, \Re \gamma>$ $0, a>0$ :

$$
\begin{aligned}
\int_{0}^{\infty} & \frac{x d x}{\gamma^{2}+x^{2}} \mathrm{e}^{-\beta x^{2}} \sin a x \\
& =-\frac{\pi}{4} \mathrm{e}^{\beta \gamma^{2}}\left[2 \sinh a \gamma+\mathrm{e}^{-a \gamma} \operatorname{erf}\left(\gamma \sqrt{\beta}-\frac{a}{2 \sqrt{\beta}}\right)-\mathrm{e}^{a \gamma} \operatorname{erf}\left(\gamma \sqrt{\beta}+\frac{a}{2 \sqrt{\beta}}\right)\right] \\
\int_{0}^{\infty} & \frac{d x}{\gamma^{2}+x^{2}} \mathrm{e}^{-\beta x^{2}} \cos a x \\
& =\frac{\pi}{4 \gamma} \mathrm{e}^{\beta \gamma^{2}}\left[2 \cosh a \gamma-\mathrm{e}^{-a \gamma} \operatorname{erf}\left(\gamma \sqrt{\beta}-\frac{a}{2 \sqrt{\beta}}\right)-\mathrm{e}^{a \gamma} \operatorname{erf}\left(\gamma \sqrt{\beta}+\frac{a}{2 \sqrt{\beta}}\right)\right]
\end{aligned}
$$


For the radial Green's function, respectively, I obtain

$$
\begin{aligned}
& \frac{\mathrm{i}}{\hbar} \int_{0}^{\infty} d T \mathrm{e}^{\mathrm{i} T E / \hbar} K_{l}^{\left(\mathcal{H}_{-1}^{(D)}\right)}\left(\tau^{\prime \prime}, \tau^{\prime} ; T\right)=\frac{1}{\hbar} \sqrt{-\frac{m}{2 E}} \exp \left(-\left|\tau^{\prime \prime}-\tau^{\prime}\right| \frac{\sqrt{-2 m R^{2} E}}{\hbar}\right) \\
& +\frac{1}{2} \sum_{n=0}^{N-1} \frac{(N-n)(2 N-n) !}{-\hbar^{2}(N-n)^{2} / 2 m R^{2}-E} \frac{1}{n !} P_{N}^{n-N}\left(\tanh \tau^{\prime}\right) P_{N}^{n-N}\left(\tanh \tau^{\prime \prime}\right) \\
& \quad \times\left\{1-\left(1-\frac{\hbar(N-n)}{\sqrt{-2 m R^{2} E}}\right) \cosh \left[\left|\tau^{\prime \prime}-\tau^{\prime}\right|\left(N-n+\frac{\sqrt{-2 m R^{2} E}}{\hbar}\right)\right]\right\} .
\end{aligned}
$$

Here use has been made of the Laplace-Fourier transformations [8], p.177:

$$
\int_{0}^{\infty} d t \mathrm{e}^{(a-p) t} \operatorname{erfc}\left(\sqrt{a t}+\frac{1}{2} \sqrt{\frac{\beta}{t}}\right)=\frac{\exp (-\sqrt{a \beta}-\sqrt{p \beta})}{\sqrt{p}(\sqrt{p}+\sqrt{a})} .
$$

Equation (30) represents the spectral expansion, where wave-functions and energy spectra can be read off.

\section{The "Kepler-Problem".}

In the path integral (28) the following potential on $\mathcal{H}_{-1}^{(D)}$ is easily incorporated:

$$
\begin{aligned}
V(\tau) & =-\frac{q^{2}}{r} \sqrt{\left(\frac{r}{R}\right)^{2}-1} \\
& =-\frac{q^{2}}{R} \tanh \tau
\end{aligned}
$$

where $r^{2}=\sum_{i=1}^{D-1} x_{i}^{2} \geq R^{2}$. For $D=4$ (34) has the structure of a Kepler-problem in a space of constant curvature [2, 18]. In our case of the single-sheeted hyperboloid we want to keep this notion for every dimension $D$, and we will see that a similar structure familiar from the usual Coulomb-problem in the energy spectrum will in fact arise, however with some significant different features. Furthermore, the potential (35) is not singular for any value of $\tau \in \mathbb{R}$.

Implementing the potential (35) in the radial path integral (28) yields

$$
\begin{aligned}
& K_{l}^{\left(q^{2}\right)}\left(\tau^{\prime \prime}, \tau^{\prime} ; T\right) \\
& =\int_{\tau\left(t^{\prime}\right)=\tau^{\prime}}^{\tau\left(t^{\prime \prime}\right)=\tau^{\prime \prime}} \mathcal{D} \tau(t) \exp \left\{\frac{\mathrm{i}}{\hbar} \int_{t^{\prime}}^{t^{\prime \prime}}\left[\frac{m}{2} R^{2} \dot{\tau}^{2}+\frac{\hbar^{2}}{2 m R^{2}} \frac{\left(l+\frac{D-3}{2}\right)^{2}-1 / 4}{\cosh ^{2} \tau}+\frac{q^{2}}{R} \tanh \tau\right] d t\right\} .
\end{aligned}
$$

Equation (36) has the form of the path integral for the Rosen-Morse potential

$$
V(x)=-\frac{B}{\cosh ^{2} x / R}+A \tanh \frac{x}{R},
$$

( $A, B, R$ constants, $x \in \mathbb{R}$ ) which has been discussed in [17, 31] by means of the path integral of the modified Pöschl-Teller potential, c.f. the Appendix. Identifying

$$
A=-\frac{q^{2}}{R}, \quad B=\hbar^{2} \frac{\left(l+\frac{D-2}{2}\right)^{2}-\frac{1}{4}}{2 m R^{2}}, \quad \tau=\frac{x}{R}
$$


gives the path integral solution

$$
\begin{aligned}
\frac{\mathrm{i}}{\hbar} & \int_{0}^{\infty} d T \mathrm{e}^{\mathrm{i} T E / \hbar} \int_{\tau\left(t^{\prime}\right)=\tau^{\prime}}^{\tau\left(t^{\prime \prime}\right)=\tau^{\prime \prime}} \mathcal{D} \tau(t) \exp \left\{\frac{\mathrm{i}}{\hbar} \int_{t^{\prime}}^{t^{\prime \prime}}\left[\frac{m}{2} R^{2} \dot{\tau}^{2}+\hbar^{2} \frac{\left(l+\frac{D-3}{2}\right)^{2}-\frac{1}{4}}{2 m R^{2} \cosh ^{2} \tau}+\frac{q^{2}}{R} \tanh \tau\right] d t\right\} \\
= & \frac{m R^{2}}{\hbar^{2}} \frac{\Gamma\left(m_{1}-L_{B}\right) \Gamma\left(L_{B}+m_{1}+1\right)}{\Gamma\left(m_{1}+m_{2}+1\right) \Gamma\left(m_{1}-m_{2}+1\right)} \\
& \times\left(\frac{1-\tanh \tau^{\prime}}{2} \cdot \frac{1-\tanh \tau^{\prime \prime}}{2}\right)^{\frac{m_{1}-m_{2}}{2}}\left(\frac{1+\tanh \tau^{\prime}}{2} \cdot \frac{1+\tanh \tau^{\prime \prime}}{2}\right)^{\frac{m_{1}+m_{2}}{2}} \\
& \times_{2} F_{1}\left(-L_{B}+m_{1}, L_{B}+m_{1}+1 ; m_{1}+m_{2}+1 ; \frac{1+\tanh \tau_{>}}{2}\right) \\
& \times{ }_{2} F_{1}\left(-L_{B}+m_{1}, L_{B}+m_{1}+1 ; m_{1}-m_{2}+1 ; \frac{1-\tanh \tau_{<}}{2}\right) \\
= & \sum_{n=0}^{N_{M}} \frac{\Psi_{n, l}^{\left(q^{2}\right) *}\left(\tau^{\prime}\right) \Psi_{n, l}^{\left(q^{2}\right)}\left(\tau^{\prime \prime}\right)}{E_{n, l}^{\left(q^{2}\right)}-E}+\int_{0}^{\infty} d p \frac{\Psi_{p, l}^{\left(q^{2}\right) *}\left(\tau^{\prime}\right) \Psi_{p, l}^{\left(q^{2}\right)}\left(\tau^{\prime \prime}\right)}{E_{p, l}^{\left(q^{2}\right)}-E} .
\end{aligned}
$$

Here are $L_{B}=l+\frac{D-4}{2}, m_{1,2}=\sqrt{m / 2} R\left(\sqrt{-q^{2} / R-E} \pm \sqrt{q^{2} / R-E}\right) / \hbar$, and $\tau_{<,>}$the smaller/ larger of $\tau^{\prime}, \tau^{\prime \prime}$, respectively. The wave-functions and the energy-spectrum are given by $[s \equiv$ $2 l+D-3, n=0, \ldots, N_{M}<l+\frac{D-4}{2}-\sqrt{R / a}$ with $a=\hbar^{2} / m q^{2}$ the Bohr radius, $k_{1}=\frac{1}{2}(1+s)$, $k_{2}=\frac{1}{2}\left[1+\frac{1}{2}(s-2 n-1)-\frac{2 m q^{2} R}{\hbar(s-2 n-1)}\right], u=\frac{1}{2}(1+\tanh \tau)$, note $\left.k_{2}-\frac{1}{2}>0\right]$ :

$$
\begin{aligned}
& \Psi_{n, l}^{\left(q^{2}\right)}(\tau)= {\left[\left(\frac{1}{R}+\frac{4 m q^{2}}{\hbar^{2}(s-2 n-1)^{2}}\right) \frac{\left(s-2 k_{2}-2 n\right) n ! \Gamma(s-n)}{\Gamma\left(s+1-n-2 k_{2}\right) \Gamma\left(2 k_{2}+n\right)}\right]^{1 / 2} 2^{n+(1-s) / 2} } \\
& \times(1-\tanh \tau)^{\frac{1}{2} s-k_{2}-n}(1+\tanh \tau)^{k_{2}-\frac{1}{2}} P_{n}^{\left(s-2 k_{2}-2 n, 2 k_{2}-1\right)}(\tanh \tau), \\
& E_{n, l}^{\left(q^{2}\right)=}-\left[\hbar^{2} \frac{\left(l+\frac{D-4}{2}-n\right)^{2}}{2 m R^{2}}+\frac{m q^{4}}{2 \hbar^{2}\left(n-l-\frac{D-4}{2}\right)^{2}}\right] .
\end{aligned}
$$

The wave-functions and the energy-spectrum of the continuous states are given by $\left[k_{2} \equiv \frac{1}{2}(1+\mathrm{i} \tilde{p})\right.$ $\left.\kappa=\frac{1}{2}(1+\mathrm{i} p), \tilde{p} \equiv \sqrt{2 m R^{2}\left(-2 q^{2} / R+\hbar^{2} p^{2} / 2 m R^{2}\right)} / \hbar>0\right]:$

$$
\begin{aligned}
& \Psi_{p, l}^{\left(q^{2}\right)}(x)= N_{p}^{\left(k_{1}, k_{2}\right)}(1-u)^{-\mathrm{i} p / 2} u^{\mathrm{i} \tilde{p} / 2} \\
& \times{ }_{2} F_{1}\left\{\frac{1}{2}[1+s+\mathrm{i}(\tilde{p}-p)], \frac{1}{2}[1-s+\mathrm{i}(\tilde{p}-p)] ; 1+\mathrm{i} \tilde{p} ; u\right\}, \\
& N_{k}^{\left(k_{1}, k_{2}\right)=} \frac{1}{\Gamma\left(2 k_{2}\right)} \sqrt{\frac{p \sinh \pi p}{2 \pi^{2}}}\left[\Gamma\left(k_{1}+k_{2}-\kappa\right) \Gamma\left(-k_{1}+k_{2}+\kappa\right)\right. \\
&\left.\times \Gamma\left(k_{1}+k_{2}+\kappa-1\right) \Gamma\left(-k_{1}+k_{2}-\kappa+1\right)\right]^{1 / 2}, \\
& E_{p, l=} \frac{\hbar^{2} p^{2}}{2 m R^{2}}-\frac{q^{2}}{R} .
\end{aligned}
$$

In the limit $q^{2}=0$ the case of $(30)$ is easily recovered. Note that for the entire problem the additional "zero-energy" shift $E_{0}^{(D)}=\hbar^{2}(D-2)^{2} / 8 m R^{2}$ has to be taken into account, c.f. (27). We see that the energy spectrum (42) of the "Kepler-problem" on the single-sheeted hyperboloid has in fact a form familar from the usual Coulomb-problem in flat space, and in spaces of (positive and negative) constant curvature [2, 18], respectively. However, in the present example the flat space limit $(R \rightarrow \infty)$ does not make any sense, and the corresponding Hilbert space does not exist. 


\section{The Constant Magnetic Field.}

Let us introduce on $\mathcal{H}_{-1}^{(3)}$ the vector-potential $\vec{A}$

$$
\vec{A}=\left(A_{\tau}, A_{\phi}\right)=\mathrm{i} B \sinh \tau(0,1) .
$$

The magnetic field is thus calculated to read as $d B=\left(\partial_{\tau} A_{\phi}-\partial_{\phi} A_{\tau}\right) d \tau \wedge d \phi=\mathrm{i} B \cosh \tau d \tau \wedge d \phi=$ $B \sqrt{\operatorname{det}\left(g_{a b}\right)}$ which has the form constant $\times$ volume-form and can therefore be interpreted as a constant field on $\mathcal{H}_{-1}^{(3)}$. Note the imaginary unit involved in $\vec{A}$ which is due to the indefinite metric of $\mathcal{H}_{-1}^{(D)}$. The path integral with the vector potential $\vec{A}$ then has the form $(b=e B / \hbar c)$

$$
\begin{aligned}
& K^{\left(\mathcal{H}_{-1}^{(3)}, b\right)}\left(\tau^{\prime \prime}, \tau^{\prime}, \phi^{\prime \prime}, \phi^{\prime} ; T\right) \\
& =\frac{1}{R^{2}} \lim _{N \rightarrow \infty}\left(\frac{m R^{2}}{2 \pi \epsilon \hbar}\right)^{N} \prod_{j=1}^{N-1} \int_{-\infty}^{\infty} \cosh \tau_{j} d \tau_{j} \int_{0}^{2 \pi} d \phi_{j} \\
& \quad \times \exp \left\{\frac{\mathrm{i}}{\hbar} \sum_{j=1}^{N}\left[\frac{m R^{2}}{2 \epsilon}\left(\Delta^{2} \tau_{j}-\widehat{\cosh }^{2} \tau_{j} \Delta^{2} \phi_{j}\right)-\mathrm{i} \hbar b \widehat{\sinh } \tau_{j} \Delta \phi_{j}-\frac{\epsilon \hbar^{2}}{8 m R^{2}}\left(1+\frac{1}{\cosh ^{2} \tau_{j}}\right)\right]\right\} \\
& \equiv \frac{1}{R^{2}} \int_{\tau\left(t^{\prime}\right)=\tau^{\prime}}^{\tau\left(t^{\prime \prime}\right)=\tau^{\prime \prime}} \cosh \tau \mathcal{D} \tau(t) \int_{\phi\left(t^{\prime \prime}\right)=\phi^{\prime \prime}} \mathcal{D} \phi(t) \\
& \quad \times \exp \left\{\frac{\mathrm{i}}{\hbar} \int_{t^{\prime}}^{t^{\prime \prime}}\left[\frac{m}{2} R^{2}\left(\dot{\tau}^{2}-\cosh ^{2} \tau \dot{\phi}^{2}\right)-\mathrm{i} \hbar b \sinh \tau \dot{\phi}-\frac{\hbar^{2}}{8 m R^{2}}\left(1+\frac{1}{\cosh ^{2} \tau}\right)\right] d t\right\} .(48)
\end{aligned}
$$

We perform a Fourier expansion according to

$$
\begin{aligned}
& K^{\left(\mathcal{H}_{-1}^{(3)}, b\right)}\left(\tau^{\prime \prime}, \tau^{\prime}, \phi^{\prime \prime}, \phi^{\prime} ; T\right)=\frac{1}{2 \pi R^{2}}\left(-\cosh \tau^{\prime} \cosh \tau^{\prime \prime}\right)^{-1 / 2} \\
& \quad \times \exp \left[-\frac{\mathrm{i} \hbar T}{2 m R^{2}}\left(b^{2}+\frac{1}{4}\right)\right] \sum_{l=-\infty}^{\infty} \mathrm{e}^{\mathrm{i} l\left(\phi^{\prime \prime}-\phi^{\prime}\right)} K_{l}^{\left(\mathcal{H}_{-1}^{(3)}, b\right)}\left(\tau^{\prime \prime}, \tau^{\prime} ; T\right), \\
& K_{l}^{\left(\mathcal{H}_{-1}^{(3)}, b\right)}\left(\tau^{\prime \prime}, \tau^{\prime} ; T\right)=\frac{1}{2 \pi} \int_{0}^{2 \pi} d \phi^{\prime \prime} \mathrm{e}^{-\mathrm{i} l\left(\phi^{\prime \prime}-\phi^{\prime}\right)} K^{\left(\mathcal{H}_{-1}^{(3)}, b\right)}\left(\tau^{\prime \prime}, \tau^{\prime}, \phi^{\prime \prime}, \phi^{\prime} ; T\right) .
\end{aligned}
$$

We therefore obtain that the radial kernel $K_{l}^{\left(\mathcal{H}_{-1}^{(3)}, b\right)}(T)$ is given by

$$
\begin{aligned}
& K_{l}^{\left(\mathcal{H}_{-1}^{(3)}, b\right)}\left(\tau^{\prime \prime}, \tau^{\prime} ; T\right) \\
& =\int_{\tau\left(t^{\prime}\right)=\tau^{\prime}}^{\tau\left(t^{\prime \prime}\right)=\tau^{\prime \prime}} \mathcal{D} \tau(t) \exp \left\{\frac{\mathrm{i}}{\hbar} \int_{t^{\prime}}^{t^{\prime \prime}}\left[\frac{m}{2} R^{2} \dot{\tau}^{2}+\frac{\hbar^{2}}{2 m R^{2}}\left(\frac{l^{2}+b^{2}-1 / 4}{\cosh ^{2} \tau}+2 \mathrm{i} l b \frac{\tanh \tau}{\cosh \tau}\right)\right] d t\right\},
\end{aligned}
$$

which is the path integral of a barrier tunneling potential $V(x)=\left(\hbar^{2} / 2 m\right)(A+B \tanh x / \cosh x+$ $C \tanh ^{2} x$ ) as discussed in [23] (compare [34] for a detailed study of reflection and scattering properties) and belongs to a class of potentials called Scarf-like potentials [6]. We perform the coordinate transformation $(1+\mathrm{i} \sinh \tau) / 2=\cosh ^{2} z$ and obtain $(M=4 m)$

$$
K_{l}^{\left(\mathcal{H}_{-1}^{(3)}, b\right)}\left(\tau^{\prime \prime}, \tau^{\prime} ; T\right) \rightarrow \int_{z\left(t^{\prime}\right)=z^{\prime}}^{z\left(t^{\prime \prime}\right)=z^{\prime \prime}} \mathcal{D} z(t) \exp \left\{\frac{\mathrm{i}}{\hbar} \int_{t^{\prime}}^{t^{\prime \prime}}\left[\frac{M}{2} \dot{z}^{2}-\frac{\hbar^{2}}{2 M}\left(\frac{C-\mathrm{i} B}{\sinh ^{2} z}-\frac{C+\mathrm{i} B}{\cosh ^{2} z}\right)\right] d s\right\}
$$




$$
\begin{aligned}
=\frac{1}{2}\left\{\sum_{n=0}^{N_{M}} \mathrm{e}^{-\mathrm{i} T E_{n}^{\left(\mathcal{H}_{-1}^{(3)}, b\right)} / \hbar} \Psi_{n}^{\left(\mathcal{H}_{-1}^{(D)}, b\right) *}\left(z^{\prime}\right) \Psi_{n}^{\left(\mathcal{H}_{-1}^{(3)}, b\right)}\left(z^{\prime \prime}\right)\right. \\
\left.\quad+\int_{-\infty}^{\infty} d p \mathrm{e}^{-\mathrm{i} T E_{p}^{\left(\mathcal{H}_{-1}^{(3)}, b\right)} / \hbar} \Psi_{p}^{\left(\mathcal{H}_{-1}^{(3)}, b\right) *}\left(z^{\prime}\right) \Psi_{p}^{\left(\mathcal{H}_{-1}^{(3)}, b\right)}\left(z^{\prime \prime}\right)\right\},
\end{aligned}
$$

We do not worry about the fact that this is a complex coordinate transformation (compare also [2] in the treatment of the Kepler problem in a space of constant positive curvature, where an even more complicated coordinate transformation has been made, accompanied by an additional timetransformation). Due to the specific nature of the vector potential we have chosen, the latter path integral is a usual one-dimensional path integral with a real potential [e.g. $C \pm \mathrm{i} B=(l \pm b)^{2}-\frac{1}{4}$ ]. Here $k_{1}=\frac{1}{2}(1+\sqrt{C+\mathrm{i} B+1 / 4})=\frac{1}{2}(1+|l+b|), k_{2}=\frac{1}{2}(1+|l-b|)$ in the notation of the Appendix (the correct signs of the square roots follow from the vanishing of the bound state wave-functions for $x \rightarrow \pm \infty$ ). Therefore we obtain for the energy spectrum for the motion $\mathcal{H}_{-1}^{(3)}$ with a constant magnetic field

$$
E_{n}^{\left(\mathcal{H}_{-1}^{(3)}, b\right)}=\frac{\hbar^{2}}{2 m R^{2}}\left[\frac{1}{4}+b^{2}-\left(n+\frac{1}{2}-\frac{1}{2}|l+b|+\frac{1}{2}|l-b|\right)^{2}\right] .
$$

We have $n=0,1,2, \ldots, N_{M}<\frac{1}{2}(|l+b|-|l-b|-1)$. For the bound state wave functions we get (reinserting $z \rightarrow \tau$ )

$$
\begin{aligned}
\Psi_{n, l}^{\left(\mathcal{H}_{-1}^{(3)}, b\right)}(\tau)= & {\left[\frac{(|l+b|-|l-b|-n-1) n ! \Gamma(|l+b|-n)}{\Gamma(|l+b|-|l-b|-n) \Gamma(|l-b|+n+1)}\right]^{\frac{1}{2}} } \\
& \times\left(\frac{\mathrm{i} \sinh \tau-1}{2}\right)^{\frac{1}{2}\left(\frac{1}{2}+|l-b|\right)}\left(\frac{\mathrm{i} \sinh \tau+1}{2}\right)^{\frac{1}{2}\left(\frac{1}{2}-|l+b|\right)} P_{n}^{(|l-b|,-|l+b|)}(\mathrm{i} \sinh \tau) .
\end{aligned}
$$

and the $P_{n}^{(a, b)}(z), z \in \mathbb{C}$, are Jacobi polynomials.

Rescaling the parameter $p$ according to $p \rightarrow 2 p$ in the $p$-integral for the continuous states we get for the continuous spectrum

$$
E_{p}^{\left(\mathcal{H}_{-1}^{(D)}, b\right)}=\frac{\hbar^{2}}{2 m R^{2}}\left(p^{2}+b^{2}+\frac{1}{4}\right)
$$

and the wave functions have the form

$$
\begin{aligned}
\Psi_{p, l}^{\left(\mathcal{H}_{-1}^{(3)}, b\right)}(\tau)= & \frac{\sqrt{p \sinh 2 \pi p}}{\pi \Gamma(1+|l-b|)}\left|\Gamma\left[\frac{1}{2}(1+|l-b|+|l+b|)-\mathrm{i} p\right] \Gamma\left[\frac{1}{2}(1+|l-b|-|l+b|)-\mathrm{i} p\right]\right| \\
& \times\left(\frac{\mathrm{i} \sinh \tau+1}{2}\right)^{\frac{1}{2}\left(\frac{1}{2}+|l-b|\right)}\left(\frac{\mathrm{i} \sinh \tau-1}{2}\right)^{\mathrm{i} p-\frac{1}{2}\left(\frac{1}{2}+|l-b|\right)} \\
& \times{ }_{2} F_{1}\left[\frac{1}{2}(1+|l-b|+|l+b|)-\mathrm{i} p\right. \\
& \left.\frac{1}{2}(1+|l-b|-|l+b|)-\mathrm{i} p ; 1+|l-b| ; \frac{\mathrm{i} \sinh \tau-1}{\mathrm{i} \sinh \tau+1}\right]
\end{aligned}
$$

\section{Summary and Discussion.}

In this note I have studied path integration on the (D-1)-dimensional single-sheeted hyperboloid in a conveniently chosen coordinate system of $(1, D-1)$-dimensional pseudo-bispherical polar coordinates: first the two-dimensional, second its higher-dimensional generalization, third a potential problem, and finally the case of a constant magnetic field. In all cases the propagators, 
the Green functions, and the corresponding wave-functions and energy spectra could be easily determined by the formalism. We found that in comparison to the (two-sheeted) pseudosphere bound states are allowed already for the free motion on the single-sheeted hyperboloid, where the number of bound stated is determined by the angular momentum number. Similarly as in the case of the pseudosphere [26], the hyperbolic plane with magnetic fields [19], and other hyperbolic spaces [22], a "zero-energy" shift $E_{0}^{(D)}=\hbar^{2}(D-2)^{2} / 8 m R^{2}$ appeared in the energy spectra. We also found that in even dimensions $D$ the corresponding "radial" propagator for the free motion has the form of a reflectionless potential propagator, a property which allows simplifications in the explicit form of the radial propagator.

The potential problem on $\mathcal{H}_{-1}^{(D)}$ which was studied, we called "Kepler-problem" on $\mathcal{H}_{-1}^{(D)}$ due to its general structure in terms of the coordinates of the embedding space. The corresponding path integral could be reduced to a known path integral solution, namely of the path integral for the Rosen-Morse potential. However, as we saw, it cannot be interpreted as a genuine Coulombproblem as known from the other spaces of constant curvature because it is not singular, it is the solution of the homogeneous Laplace equation (and not of the inhomogeneous one), and the flat space limit does not make sense.

In the forth Section we discussed the case of constant magnetic field on the two-dimensional single-sheeted hyperboloid. Here another path integral identity came into play, i.e. the path integral solution from a specific form of a Scarf-like potential, respectively a hyperbolic barrier potential.

In all our problems, free motion, "Kepler-problem", and the constant magnetic field, we could observe a nice interplay between motion in spaces of constant curvature on the one side, and potential problems emerging from them by separating the angular variables on the other. This feature is well-known also from other realizations of (real) Lobachevskian spaces [19, 20, 22]. It has its origin in the underlying group structure of the space in question, respectively the corresponding dynamical group of the potential problem [28, 29], where the most known example is the Hydrogen atom in flat with its $\mathrm{O}(4)$ symmetry. The pseudo-bispherical coordinates coming from the $\mathrm{SO}(1, D-1)$ group structure of $\mathcal{H}_{-1}^{(D)}$ allow the separation of the angular variables due to $\mathrm{SO}(m, n) \supset \mathrm{SO}(m) \times \mathrm{SO}(n)$, and the remaining path radial- (i.e. $\tau$-) path integration can be transformed into a $\mathrm{SU}(1,1)$ path integration.

From the present model no quantum mechanical discussion seems to have been made until now, an operator approach as well as a path integral approach. The solution of path integration on $\mathcal{H}_{-1}^{(D)}$, together with the potential problem and the case of a magnetic field, has in comparison to an operator approach the advantage of presenting a global picture of the quantum theory in question, whereas the Schrödinger approach allows only a local one, and the explicit form of the Feynman kernel gives the complete solution in terms of the wave-functions and the energyspectrum, respectively. The examples demonstrate once more the consistency as well as the universal utility and feasibility of the Feynman path integral and of our general method developed in [25].

\section{Acknowledgement.}

I would like to thank E.Rabinovici for drawing my attention to the problem of the single-sheeted hyperboloid.

\section{Appendix.}

In this Appendix we cite an important path integral identity important for the discussion in the text. Let us consider quantum mechanical models related to the modified Pöschl-Teller (mPT) 
potential

$$
V^{(m P T)}(\tau)=\frac{\hbar^{2}}{2 m}\left(\frac{\eta^{2}-\frac{1}{4}}{\sinh ^{2} \tau}-\frac{\nu^{2}-\frac{1}{4}}{\cosh ^{2} \tau}\right), \quad \tau>0,
$$

which has a (hidden) SU(1,1) symmetry. The path integral solution is due to [3, 30] and has the form (we use the notation of Ref. [10] for the bound and continuous states, respectively, $k_{1}=\frac{1}{2}(1 \pm \nu), k_{2}=\frac{1}{2}(1 \pm \eta)$, for the explicit form of the Green function compare [23, 31] )

$$
\begin{aligned}
& \frac{\mathrm{i}}{\hbar} \int_{0}^{\infty} d T \mathrm{e}^{\mathrm{i} T E / \hbar} \int_{r\left(t^{\prime}\right)=r^{\prime}}^{r\left(t^{\prime \prime}\right)=r^{\prime \prime}} \mathcal{D} r(t) \exp \left\{\frac{\mathrm{i}}{\hbar} \int_{t^{\prime}}^{t^{\prime \prime}}\left[\frac{m}{2} \dot{r}^{2}-\frac{\hbar^{2}}{2 m}\left(\frac{\eta^{2}-\frac{1}{4}}{\sinh ^{2} r}-\frac{\nu^{2}-\frac{1}{4}}{\cosh ^{2} r}\right)\right] d t\right\} \\
& =\frac{m}{\hbar^{2}} \frac{\Gamma\left(m_{1}-L_{\nu}\right) \Gamma\left(L_{\nu}+m_{1}+1\right)}{\Gamma\left(m_{1}+m_{2}+1\right) \Gamma\left(m_{1}-m_{2}+1\right)} \\
& \quad \times\left(\cosh r^{\prime} \cosh r^{\prime \prime}\right)^{-\left(m_{1}-m_{2}\right)}\left(\tanh r^{\prime} \tanh r^{\prime \prime}\right)^{m_{1}+m_{2}+1 / 2} \\
& \quad \times_{2} F_{1}\left(-L_{\nu}+m_{1}, L_{\nu}+m_{1}+1 ; m_{1}-m_{2}+1 ; \frac{1}{\cosh ^{2} r_{<}}\right) \\
& \quad \times_{2} F_{1}\left(-L_{\nu}+m_{1}, L_{\nu}+m_{1}+1 ; m_{1}+m_{2}+1 ; \tanh ^{2} r_{>}\right) \\
& =\sum_{n=0}^{N_{M}} \frac{\Psi_{n}^{\left(k_{1}, k_{2}\right) *}\left(r^{\prime}\right) \Psi_{n}^{\left(k_{1}, k_{2}\right)}\left(r^{\prime \prime}\right)}{E_{n}-E}+\int_{0}^{\infty} d p \frac{\Psi_{p}^{\left(k_{1}, k_{2}\right) *}\left(r^{\prime}\right) \Psi_{p}^{\left(k_{1}, k_{2}\right)}\left(r^{\prime \prime}\right)}{\hbar^{2} p^{2} / 2 m-E},
\end{aligned}
$$

$\left(m_{1,2}=\frac{1}{2}\left(\eta \pm \sqrt{-2 m E} / \hbar, L_{\nu}=\frac{1}{2}(1-\nu)\right.\right.$. The correct signs depend on the boundary conditions for $r \rightarrow 0$ and $r \rightarrow \infty$, respectively. Here we have introduced the Green function

$$
G\left(q^{\prime \prime}, q^{\prime} ; E\right):=\frac{\mathrm{i}}{\hbar} \int_{0}^{\infty} d T \mathrm{e}^{\mathrm{i}(E+\mathrm{i} \epsilon) T / \hbar} K\left(q^{\prime \prime}, q^{\prime} ; T\right)=\left\langle q^{\prime \prime}\left|\frac{1}{H-E-\mathrm{i} \epsilon}\right| q^{\prime}\right\rangle,
$$

( $\underline{H}$ the Hamiltonian) where a small positive imaginary part $(\epsilon>0)$ has been added to the energy $E$. (We shall not explicitly write the $\mathrm{i} \epsilon$, but will tacitly assume that the various expressions are regularized according to this rule). The bound state wave-functions are e.g. given by ( $\kappa=$ $\left.k_{1}-k_{2}-n\right)$

$$
\begin{aligned}
& \Psi_{n}^{\left(k_{1}, k_{2}\right)}(\tau)=\frac{1}{\Gamma\left(2 k_{2}\right)}\left[\frac{2(2 \kappa-1) \Gamma\left(k_{1}+k_{2}-\kappa\right) \Gamma\left(k_{1}+k_{2}+\kappa-1\right)}{\Gamma\left(k_{1}-k_{2}+\kappa\right) \Gamma\left(k_{1}-k_{2}-\kappa+1\right)}\right]^{1 / 2} \\
& \times(\sinh \tau)^{2 k_{2}-\frac{1}{2}}(\cosh \tau)^{-2 k_{1}+3 / 2} \\
& \times_{2} F_{1}\left(-k_{1}+k_{2}+\kappa,-k_{1}+k_{2}-\kappa+1 ; 2 k_{2} ;-\sinh ^{2} \tau\right) \text {, } \\
& E_{n}=-\frac{\hbar^{2}}{2 m}\left[2\left(k_{1}-k_{2}-n\right)-1\right]^{2} \text {. }
\end{aligned}
$$

Here is $n=0,1, \ldots, N_{m}<k_{1}-k_{2}-1 / 2$, with $N_{M}$ the maximum number of bound states. The continuous states have the form $\left[\kappa=\frac{1}{2}(1+\mathrm{i} p)\right]$

$$
\begin{aligned}
& \Psi_{p}^{\left(k_{1}, k_{2}\right)}(\tau)= N_{p}^{\left(k_{1}, k_{2}\right)}(\cosh \tau)^{\kappa-2 k_{2}-1 / 2}(\sinh \tau)^{2 k_{2}-1 / 2} \\
& \times{ }_{2} F_{1}\left(k_{1}+k_{2}-\kappa, k_{2}-k_{1}-\kappa+1 ; 2 k_{2} ; \tanh ^{2} \tau\right) \\
& N_{p}^{\left(k_{1}, k_{2}\right)=} \frac{1}{\Gamma\left(2 k_{2}\right)} \sqrt{\frac{p \sinh \pi p}{2 \pi^{2}}}\left[\Gamma\left(k_{1}+k_{2}-\kappa\right) \Gamma\left(-k_{1}+k_{2}+\kappa\right)\right. \\
&\left.\times \Gamma\left(k_{1}+k_{2}+\kappa-1\right) \Gamma\left(-k_{1}+k_{2}-\kappa+1\right)\right]^{1 / 2}
\end{aligned}
$$


and $E_{p}=\hbar^{2} p^{2} / 2 m$. Here the functions ${ }_{2} F_{1}(a, b ; c ; z)(z \in \mathbb{C})$ denote hypergeometric functions.

Of particular importance is the following special case, where a path integral solution according to [1], 20, 31] has the form $\left(n=0,1, \ldots, N_{M}<l-\frac{1}{2}, l>0, x \in \mathbb{R}\right)$

$$
\begin{aligned}
& \frac{\mathrm{i}}{\hbar} \int_{0}^{\infty} d T \mathrm{e}^{\mathrm{i} T E / \hbar} \int_{x\left(t^{\prime}\right)=x^{\prime}}^{x\left(t^{\prime \prime}\right)=x^{\prime \prime}} \mathcal{D} x(t) \exp \left[\frac{\mathrm{i}}{\hbar} \int_{t^{\prime}}^{t^{\prime \prime}}\left(\frac{m}{2} \dot{x}^{2}+\frac{\hbar^{2}}{2 m} \frac{l^{2}-\frac{1}{4}}{\cosh ^{2} x}\right) d t\right] \\
&= \frac{m}{\hbar^{2}} \Gamma\left(\frac{1}{\hbar} \sqrt{-2 m E}-l+\frac{1}{2}\right) \Gamma\left(\frac{1}{\hbar} \sqrt{-2 m E}+l+\frac{1}{2}\right) \\
& \times P_{l-1 / 2}^{-\sqrt{-2 m E} / \hbar}\left(\tanh x_{<}\right) P_{l-1 / 2}^{-\sqrt{-2 m E} / \hbar}(-\tanh x) \\
&= \sum_{n=0}^{N_{M}}\left(n-l-\frac{1}{2}\right) \frac{\Gamma(2 l-n)}{n !} \frac{P_{l-1 / 2}^{n-l+\frac{1}{2}}\left(\tanh x^{\prime}\right) P_{l-1 / 2}^{n-l+\frac{1}{2}}\left(\tanh x^{\prime \prime}\right)}{-\hbar^{2}\left(n-l+\frac{1}{2}\right)^{2} / 2 m-E} \\
& \quad+\frac{1}{2} \int_{-\infty}^{\infty} \frac{d p p \sinh \pi p}{\hbar^{2} p^{2} / 2 m-E} \frac{P_{l-1 / 2}^{\mathrm{i} p}\left(\tanh x^{\prime}\right) P_{l-1 / 2}^{-\mathrm{i} p}\left(\tanh x^{\prime \prime}\right)}{\cos ^{2} \pi l+\sinh ^{2} \pi p} .
\end{aligned}
$$

Here $P_{\nu}^{\mu}(x)$ are Legendre functions of the first kind.

\section{References}

[1] A.Anderson and S.B.Anderson: Phase Space Path Integration of Integrable Quantum Systems; Ann.Phys.(N.Y.) 199 (1990) 155

[2] A.O.Barut, A.Inomata and G.Junker: Path Integral Treatment of the Hydrogen Atom in a Curved Space of Constant Curvature; J.Phys.A: Math.Gen. 20 (1987) 6271; Path Integral Treatment of the Hydrogen Atom in a Curved Space of Constant Curvature II; J.Phys.A: Math.Gen. 23 (1990) 1179

[3] M.Böhm and G.Junker: The SU(1,1) Propagator as a Path Integral Over Noncompact Groups; Phys.Lett. A 117 (1986) 375; Path Integration Over Compact and Noncompact Rotation Groups; J.Math.Phys. 28 (1987) 1978

[4] R.E.Crandall: Exact Propagator for Reflectionless Potentials; J.Phys.A: Math. Gen. 16 (1983) 3005; R.E.Crandall and B.R.Litt: Reassembly and Time Advance in Reflectionless Scattering; Ann.Phys. (N.Y.) 146 (1983) 458

[5] B.S.DeWitt: Dynamical Theory in Curved Spaces. I. A Review of the Classical and Quantum Action Principles; Rev.Mod.Phys. 29 (1957) 377

[6] R.Dutt, A.Khare and U.P.Sukhatme: Supersymmetry, Shape Invariance, and Exactly Solvable Potentials; Amer.J.Phys. 56 (1988) 163;

R.De, R.Dutt and U.Sukhatme: Mapping of Shape Invariant Potentials Under Point Canonical Transformations; J.Phys.A: Math.Gen. 25 (1992) L 843; Path-Integral Solutions for Shape-Invariant Potentials Using Point Canonical Transformations; Phys.Rev. A 46 (1992) 6869

[7] A.Erdélyi, W.Magnus, F.Oberhettinger and F.G.Tricomi (Eds.): Higher Transcendental Functions, Vol.II (McGraw Hill, New York, 1955)

[8] A.Erdélyi, W.Magnus, F.Oberhettinger and F.G.Tricomi (Eds.): Tables of Integral Transforms, Vol.I (McGraw Hill, New York, 1954)

[9] R.P.Feynman: Space-Time Approach to Non-Relativistic Quantum Mechanics; Rev.Mod.Phys. 20 (1948) 367

[10] A.Frank and K.B.Wolf: Lie Algebras for Systems With Mixed Spectra.I. The Scattering Pöschl-Teller Potential; J.Math.Phys. 25 (1985) 973. 
[11] I.M.Gel'fand and M.I.Graev: Geometry of Homogeneous Spaces, Representations of Groups in Homogeneous Spaces and Related Questions. I; Amer.Math.Soc.Transl., Ser.2 37 (1964) 351

[12] I.M.Gel'fand, M.I.Graev, and N.Ya.Vilenkin: Generalized Functions, Vol.5 (Academic Press, New York, 1966)

[13] J.-L.Gervais and A.Jevicki: Point Canonical Transformations in the Path Integral; Nucl.Phys. B 110 (1976) 93

[14] I.S.Gradshteyn and I.M.Ryzhik: Table of Integrals, Series, and Products (Academic Press, New York, 1980)

[15] M.B.Green, J.H.Schwarz, and E.Witten: Superstring Theory I\&II. Cambridge: Cambridge University Press, 1988

[16] C.Grosche: The Product Form for Path Integrals on Curved Manifolds; Phys.Lett. A 128 (1988) 113

[17] C.Grosche: Path Integral Solution of a Class of Potentials Related to the Pöschl-Teller Potential; J.Phys.A: Math.Gen. 22 (1989) 5073

[18] C.Grosche: The Path Integral for the Kepler Problem on the Pseudosphere; Ann.Phys.(N.Y.) 204 (1990) 208

[19] C.Grosche: Path Integration on the Hyperbolic Plane With a Magnetic Field; Ann.Phys.(N.Y.) 201 (1990) 258

[20] C.Grosche: The Path Integral on the Poincaré Disc, the Poincaré Upper Half-Plane and on the Hyperbolic Strip; Fortschr.Phys. 38 (1990) 531

[21] C.Grosche: Separation of Variables in Path Integrals and Path Integral Solution of Two Potentials on the Poincaré Upper Half-Plane; J.Phys.A: Math.Gen. 23 (1990) 4885

[22] C.Grosche: Path Integration on Hyperbolic Spaces; J.Phys.A: Math.Gen. 25 (1992) 4211

[23] C.Grosche: Path Integral Solution of Scarf-Like Potentials; Trieste preprint, SISSA/179/92/FM, October 1992

[24] C.Grosche and F.Steiner: The Path Integral on the Poincaré Upper Half Plane and for Liouville Quantum Mechanics; Phys.Lett. A 123 (1987) 319

[25] C.Grosche and F.Steiner: Path Integrals on Curved Manifolds; Zeitschr.Phys. C 36 (1987) 699

[26] C.Grosche and F.Steiner: The Path Integral on the Pseudosphere; Ann.Phys.(N.Y.) 182 (1988) 120

[27] C.Grosche and F.Steiner: Table of Feynman Path Integrals; to appear in: Springer Tracts in Modern Physics

[28] A.Inomata: Roles of Dynamical Groups in Path Integration; to appear in the proceedings of the "International Workshop on 'Symmetry Methods in Physics' in Memory of Prof. Ya. A. Smorodinsky", Dubna, July 1993

[29] A.Inomata, H.Kuratsuji and C.C.Gerry: Path Integrals and Coherent States of SU(2) and SU(1,1) (World Scientific, Singapore, 1992)

[30] A.Inomata and R.Wilson: Path Integral Realization of a Dynamical Group; Lecture Notes in Physics 261, p.42 (Springer-Verlag, Berlin, 1985).

[31] H.Kleinert and I.Mustapic: Summing the Spectral Representations of Pöschl-Teller and Rosen-Morse Fixed-Energy Amplitudes; J.Math.Phys. 33 (1992) 643

[32] L.D.Landau and E.M.Lifschitz: Lehrbuch der Theoretischen Physik, Vol.III (Akademie Verlag, Berlin, 1979)

[33] N.K.Pak and I.Sökmen: General New-Time Formalism in the Path Integral; Phys.Rev. A 30 (1984) 1629

[34] D.Pertsch: Exact Solution of the Schrödinger Equation for a Potential Well with Barrier and Other Potentials; J.Phys.A: Math.Gen. 23 (1990) 4145

[35] N.Ja.Vilenkin: Special Functions Connected with Class 1 Representations of Groups of Motions in Spaces of Constant Curvature; Trans.Moscow Math.Soc. (1963) 209 